\title{
Accès gratuit à la Cochrane Library
}

Hermann Amstada, Erik von Elmb

a Dr med., Secrétaire général de I'ASSM; ' Dr med., Co-Directeur Cochrane Suisse

\author{
L'Académie Suisse des Sciences Médicales (ASSM) s'engage depuis des années pour \\ offrir aux médecins un accès à la Cochrane Library et leur permettre ainsi de main- \\ tenir leurs connaissances à jour. Depuis le début de l'année, grâce à une licence \\ nationale, l'accès à la Cochrane Library est gratuit; le nombre des utilisateurs a déjà \\ plus que doublé.
}

Ce qui, dans la prise en charge médicale, était encore considéré comme juste hier, est aujourd'hui dépassé. Nous ne sommes pas à l'abri d'erreurs. Les saignées pratiquées pendant des siècles nous apparaissent aujourd'hui comme une erreur évidente qui a probablement coûté la vie à de nombreux malades. Des études coûteuses sont souvent nécessaires pour vérifier si, au final, une mesure médicale - qu'elle soit établie ou innovante - est bénéfique ou dommageable. Rappelons-nous les longs débats autour de la thérapie hormonale substitutive il y a quelques années.

Jusqu'alors, les praticiens ne pouvaient pas accéder aisément et rapidement aux connaissances scientifiques les plus récentes. Si, grâce à «Open Access», de nombreuses informations de qualité sont accessibles gratuitement, une grande partie de la littérature médicale actuelle reste néanmoins réservée aux abonnés ou aux titulaires de licences et, compte tenu des tarifs, l'accès est souvent limité aux institutions académiques. Ainsi, il arrive que des années passent jusqu'à ce que des résultats de la recherche clinique se traduisent en améliorations dans la pratique. C'est ce que l'on appelle le «know-do gap».

\section{Preuve scientifique pour la pratique}

Dès 2009 l'ASSM exigeait, dans sa feuille de route $L a$ médecine comme science, que les jeunes médecins puissent garder le contact avec la science pendant leur formation postgraduée. Non seulement les médecins des hôpitaux universitaires, mais également ceux des plus petits hôpitaux ou les praticiens en cabinets privés devraient être en mesure de soigner leurs patients sur la base des données les plus récentes - au sens de la «médecine fondée sur les preuves». Les médecins de premier recours suisses révèlent, dans une enquête récente, les difficultés qu'ils rencontrent pour travailler sur la base de preuves scientifiques (Bengough et al. BMC Fam Pract 2015). L'accès souvent insuffisant aux revues spécialisées et aux banques de données en est l'une des principales raisons. Par ailleurs, le manque de temps et la collaboration du patient - jugée moindre dans le secteur ambulatoire - jouent également un rôle important.

Les connaissances les plus récentes de la science représentent donc l'un des principaux piliers de la prise en charge médicale basée sur les preuves scientifiques; à condition toutefois qu'elles puissent être conciliées avec les priorités des patients et des soignants.

\section{Les revues Cochrane comme standards de qualité}

Depuis plus de 20 ans, le réseau mondial «Cochrane» établit des revues systématiques qui regroupent les résultats de la recherche concernant des questions clairement définies, constituant ainsi une source d'informations fiables pour une prise en charge médicale basée sur des preuves scientifiques. Actuellement plus de 6800 revues Cochrane sont disponibles; elles sont reconnues au niveau international comme standards de qualité et reflètent l'état de la science quant à l'efficacité et aux effets secondaires des interventions thérapeutiques et préventives.

Une étude récente a analysé la qualité des rapports des revues systématiques. A cet égard, toutes les études indexées dans Medline en février 2014 ont fait l'objet d'une évaluation quant à leur intégralité (Page et al. PLoS Med 2016). En moyenne, les revues de Cochrane étaient plus complètes que les autres revues systématiques. Ceci s'explique par les standards internes appliqués à tous les groupes de revues Cochrane qui sont vérifiés avant la publication des revues nouvelles ou actualisées. De nombreuses revues Cochrane servent alors de base pour l'élaboration de directives et de rapports HTA.

Ainsi, sont fournies des informations de santé dont l'évaluation critique et la synthèse ont été réalisées selon des méthodes reconnues et sans aucune influence des sponsors commerciaux. Ceci permet un gain de 
temps appréciable dans la pratique clinique, mais profite également aux décisionnaires du système de santé et aux patients intéressés.

\section{Le réseau international Cochrane}

Environ 37000 personnes contribuent aux activités de Cochrane dans plus de 130 pays. L'engagement s'étend de l'élaboration de revues Cochrane jusqu'à la participation aux traductions de résumés ou à l'identification d'études randomisées dans le cadre du projet «Cochrane Crowd».

Dans la plupart des pays, les centres nationaux Cochrane sont implantés dans des institutions académiques et médicales. Cochrane Suisse a été fondée en 2010 et est basée à l'Institut universitaire de médecine sociale et préventive (IUMSP) à Lausanne. L'une de ses activités prioritaires consiste à soutenir et développer les revues systématiques concernant les interventions de santé publique basées sur la population. En collaboration avec quatre autres centres universitaires en Suisse, en Allemagne et en Autriche, un satellite européen du groupe de revues "Cochrane Public Health" a été fondé; le siège de se groupe est situé à Melbourne.

\section{Une licence nationale de la Cochrane Library pour la Suisse}

L'Académie Suisse des Sciences Médicales (ASSM) s'engage depuis des années pour que les professionnels de la santé puissent accéder gratuitement à la Cochrane Library et que leurs connaissances restent à jour une fois leur formation pré- et postgraduée achevée. C'est pourquoi l'ASSM s'est mobilisée pour obtenir un accès gratuit à la Cochrane Library dans le cadre d'une licence

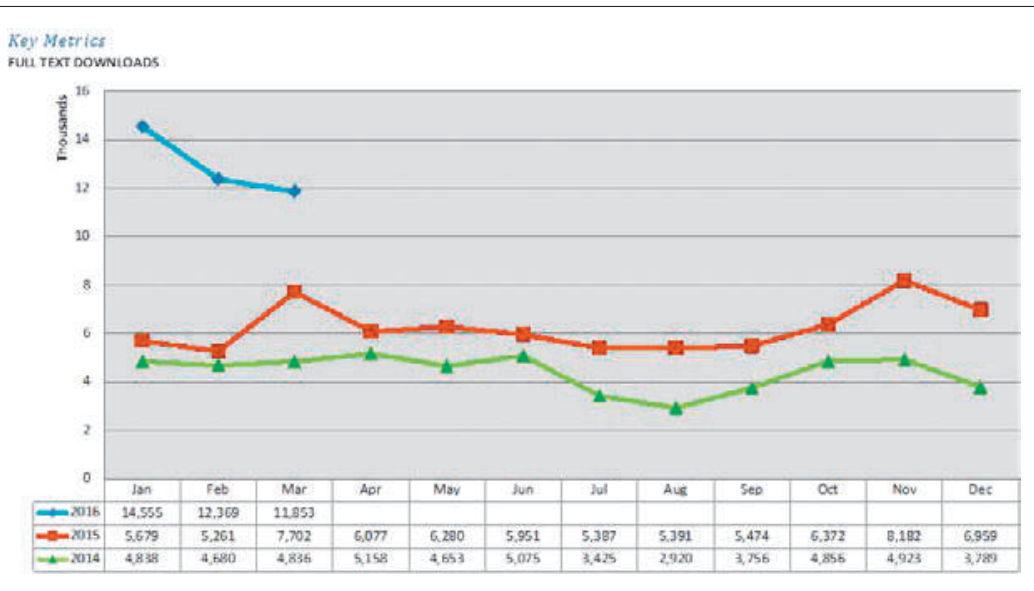

Cochrane Library

SAMW Impact and Usage report 2016 Q1 (Jan-Mar)

38777 full text downloads of articles from the Cochrane Library were recorded on Wiley Online Library in 01 2016; this figure is 108\% higher than the same time period in 2015. nationale. Cette licence nationale, co-financée par l'Office fédéral de la santé publique et les bibliothèques universitaires, permet, depuis le début de cette année, d'accéder gratuitement à cette banque de données depuis chaque ordinateur suisse. Dans un premier temps, le contrat est conclu pour la période de 2016 à 2020.

Outre les versions intégrales des revues Cochrane, cinq autres banques de données font partie de la Cochrane Library. On dispose, entre autres, de vastes collections de références d'études randomisées (CENTRAL), d'autres revues systématiques (DARE) et d'études d'économie de la santé. L'équipe de Cochrane Suisse se tient à disposition pour toute question.

\section{Le nombre d'utilisateurs doublé depuis janvier 2016}

La nette augmentation du nombre de connexions à la Cochrane Library depuis le début de l'année, en Suisse, confirme que la faible utilisation des preuves scientifiques à disposition peut être attribuée au «Paywall» (c'est-à-dire à la barrière d'accès par une licence payante). Au cours du $1^{\text {er }}$ trimestre 2016, comparé à la même période de l'année passée, le nombre d'utilisateurs a plus que doublé (cf. fig.). En moyenne, toutes les trois minutes une revue Cochrane a été téléchargée, ce qui représente un total de 38777 documents. En comparaison avec dix pays qui proposent une licence nationale de la Cochrane Library, la Suisse se situe en quatrième position après l'Angleterre, l'Australie et l'Inde. Les revues Cochrane les plus recherchées par les utilisateurs suisses traitent de thèmes tels que l'efficacité des exercices en cas de douleurs dorsales chroniques non spécifiques, l'exactitude des Mini-Mental-Test de Folstein pour l'identification de la démence, l'efficacité de l'oxygénothérapie pour le traitement de la migraine et des algies vasculaires de la face ainsi que des fibres alimentaires pour la prévention des maladies cardiovasculaires. Il s'agit donc de thèmes qui intéressent non seulement les professionnels de la santé, mais également toute personne qui se pose des questions sur sa santé. Pour faciliter l'utilisation de la Cochrane Library, un flyer avec le "top 10 des recommandations" et des explications simples sur la structure de la banque de données a été rédigé. Il est disponible en français sur le site web de l'ASSM sous www.samw.ch/Projets.

\section{Liens}

Cochrane Library: www.cochranelibrary.com

Cochrane Suisse: http://suisse.cochrane.org/fr/home Cochrane Crowd: http://crowd.cochrane.org

Cochrane Public Health: http://ph.cochrane.org

Cochrane Données probandes: http://www.cochrane.org/fr/evidence 